\title{
Apresentação atípica na Síndrome de Sézary com face leonina durante síndrome gripal por COVID-19
}

\author{
Atypical presentation in Sézary syndrome with leonine face during flu syndrome by COVID-19 \\ Presentación atípica en el síndrome de Sézary con cara de leonina durante el síndrome gripal por \\ COVID-19
}

Recebido: 10/03/2021 | Revisado: 17/03/2021 | Aceito: 20/03/2021 | Publicado: 27/03/2021

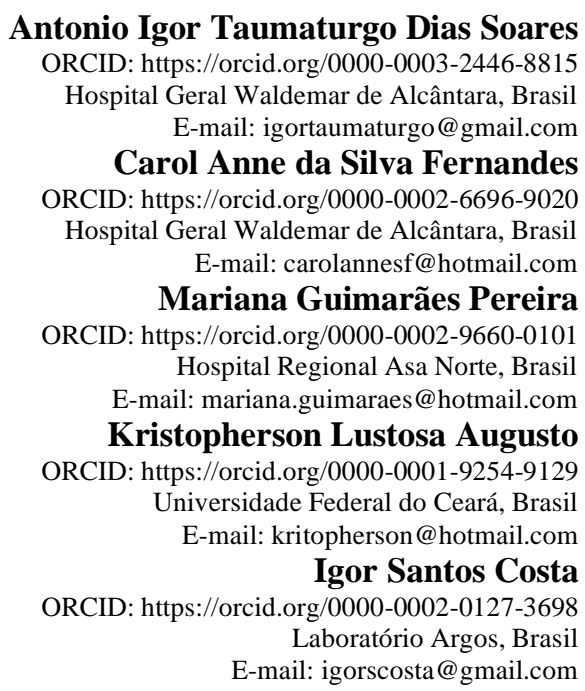

\begin{abstract}
Resumo
Os linfomas cutâneos de células T (LCCT) compreendem um grupo heterogêneo de Linfomas Não Hodgkin que se apresentam comumente como micose fungóide, de curso indolente, e uma forma leucêmica, com evolução mais agressiva, conhecida como síndrome Sézary. Essas neoplasias tornam o indivíduo mais susceptível a infecções decorrentes da imunossupressão. Em março de 2020, deflagrou-se a pandemia pelo novo coronavírus (COVID-19), que rapidamente se disseminou e causou numerosas mortes. O presente estudo descreve um caso de difícil diagnóstico representado lesões eritêmato-descamativas em tronco e membros, associada à prurido intenso em curso por oito meses, conduzida até então como farmacodermia, que evoluiu durante a internação, após infecção viral por COVID19 intra-hospitalar, com infiltrado na face, rarefação de sobrancelhas e cabelos, desfigurando a paciente, semelhante à face leonina. Paciente cursou com síndrome gripal pelo novo coronavírus, permanecendo quatorze dias em isolamento respiratório, evoluindo sem complicações graves e conduzida, em seguida, à serviço oncológico especializado.
\end{abstract}

Palavras chave: Fácies leoninas; Sézary; COVID-19.

\begin{abstract}
Cutaneous T-cell lymphomas (LCCT) comprise a heterogeneous group of Non-Hodgkin's Lymphomas that commonly present as mycosis fungoides, of indolent course, and a leukemic form, with more aggressive evolution, known as Sézary syndrome. These neoplasms make the individual more susceptible to infections resulting from immunosuppression. In March 2020, the pandemic broke out due to the new coronavirus (COVID-19), which quickly spread and caused numerous deaths. The present study describes a case of difficult diagnosis represented erythematous-desquamative lesions in the trunk and limbs, associated with intense itching in course for eight months, conducted until then as pharmacoderma, which evolved during hospitalization, after viral infection by COVID-19 intra- hospital, with infiltration in the face, thinning of eyebrows and hair, disfiguring the patient, similar to the leonine face. The patient had a flu-like syndrome due to the new coronavirus, remaining in respiratory isolation for fourteen days, progressing without serious complications and then being referred to a specialized cancer service.
\end{abstract}

Keywords: Leonine facies; Sézary; COVID-19.

\section{Resumen}

Los linfomas cutáneos de células T (LCCT) comprenden un grupo heterogéneo de linfomas no Hodgkin que comúnmente se presentan como micosis fungoide, de curso indolente, y una forma leucémica, de evolución más 
agresiva, conocida como síndrome de Sézary. Estas neoplasias hacen que el individuo sea más susceptible a infecciones resultantes de la inmunosupresión. En marzo de 2020, estalló la pandemia debido al nuevo coronavirus (COVID-19), que se propagó rápidamente y provocó numerosas muertes. El presente estudio describe un caso de difícil diagnóstico representado por lesiones eritematoso-descamativas en tronco y extremidades, asociadas a prurito intenso en curso durante ocho meses, realizado hasta entonces como farmacodermia, que evolucionó durante la hospitalización, tras infección viral por COVID-19 intra- hospital, con infiltración en el rostro, adelgazamiento de cejas y cabello, desfigurando al paciente, similar al rostro leonino. El paciente presentaba un síndrome gripal por el nuevo coronavirus, permaneciendo en aislamiento respiratorio durante catorce días, evolucionando sin complicaciones graves y siendo posteriormente derivado a un servicio oncológico especializado.

Palabras llave: Facies leonina; Sézary; COVID-19.

\section{Introdução}

Os linfomas cutâneos de células T (LCCT) compreendem um grupo, heterogêneo de Linfomas Não Hodgkin (LNH) extranodais primários, que surgem da transformação maligna de células T pós-tímicas maduras (Bologna, Jorizzo, Rapini, 2012). Dentre os LCCT's, os mais comuns são a micose fungóide (MF) de curso indolente e a síndrome de Sézary (SS), forma leucêmica, geralmente associada a um curso mais agressivo (Bologna, Jorizzo, Rapini, 2012; Willemze, Cerroni, Kempf, et al., 2019).

A incidência dos LCCT's está aumentando e, atualmente, são 6,4 casos por milhão de pessoas. A média de idade no diagnóstico é em torno dos 50 anos e a prevalência aumenta em quatro vezes naqueles acima de 70 anos (Wilcox, 2017).

A SS é a variante leucêmica do LCCT, descrita inicialmente em 1938, que se manifesta com eritrodermia, linfadenopatia e alta população de células $\mathrm{T}$ neoplásicas (células de Sézary) na pele, linfonodos e sangue periférico. Os critérios recomendados pela Sociedade Internacional de Linfomas Cutâneos (SILC) para o diagnóstico de SS integram critérios clínicos, histopatológicos, imunofenotipagem e estudos moleculares (Willemze, Cerroni, Kempf, et al., 2019; Wilcox, 2017).

Em março de 2020, a infecção pelo novo coronavírus (COVID-19) rapidamente se alastrou para diferentes regiões, sendo declarada pela Organização Mundial da Saúde (OMS) como uma pandemia. Apresentando-se como uma doença respiratória em que os sintomas podem ser leves, como um resfriado, ou graves, como a Síndrome Respiratória Aguda Grave (SARS-CoV), podendo evoluir ao óbito. Grupos que apresentam maior risco de desenvolver a doença ou apresentar a forma mais grave são idoso, portadores de doenças crônicas, imunossuprimidos e gestantes de risco (Bologna, Jorizzo, Rapini, 2012; Wang, Hu, Hu, Zhu, Liu, Zhang, et al., 2020).

Existem dados limitados sobre hospedeiros imunossuprimidos, mas relatórios publicados na China sobre os resultados de pacientes com câncer infectados com COVID-19 relatam um risco 3,5 vezes maior de necessitar de ventilação mecânica ou admissão ou morte na UTI em comparação com pacientes sem câncer (Lianget al., 2020).

O presente estudo tem por objetivo descrever um caso de Síndrome Sézary com fácies leoninas de difícil diagnóstico, antecedido por um prurido intenso, conduzido por oito meses como reação medicamentosa, que durante internação para elucidação diagnóstica, infectou-se pelo COVID-19 com sintomas gripais leves, sem complicações respiratórias e sem piora do quadro oncológico.

\section{Metodologia}

As informações contidas neste relato de caso foram obtidas por meio de entrevista com o paciente, revisão de prontuário e registros fotográficos, os quais foram todos autorizados pelo paciente por meio de termo de consentimento livre e esclarecido assinados pelo mesmo. 


\section{Relato de Caso}

Paciente, 39 anos, sexo feminino, proveniente de Quixeramobim-Ceará, admitida em um hospital público em Messejana, Ceará, Brasil, em abril de 2020, por um quadro clínico, iniciado há oito meses, com prurido e lesões cutâneas. Conduzida inicialmente como reação medicamentosa na cidade de origem, com admistração de anti-histamínicos e corticoterapia. Paciente negava doenças prévias ou história familiar semelhante. Ao exame físico, apresentava infiltração edematosa em face, alopecia, madarose semelhante a fácies leoninas (Figura 1), nódulos retroauriculares dolorosos com drenagem de secreção purulenta, eritrodermia esfoliativa e distrofia ungueal em pododáctilos (Figura 2). Linfonodos cervical e axilares $(<1 \mathrm{~cm})$.

Figura1: Fácies leoninas na SS.

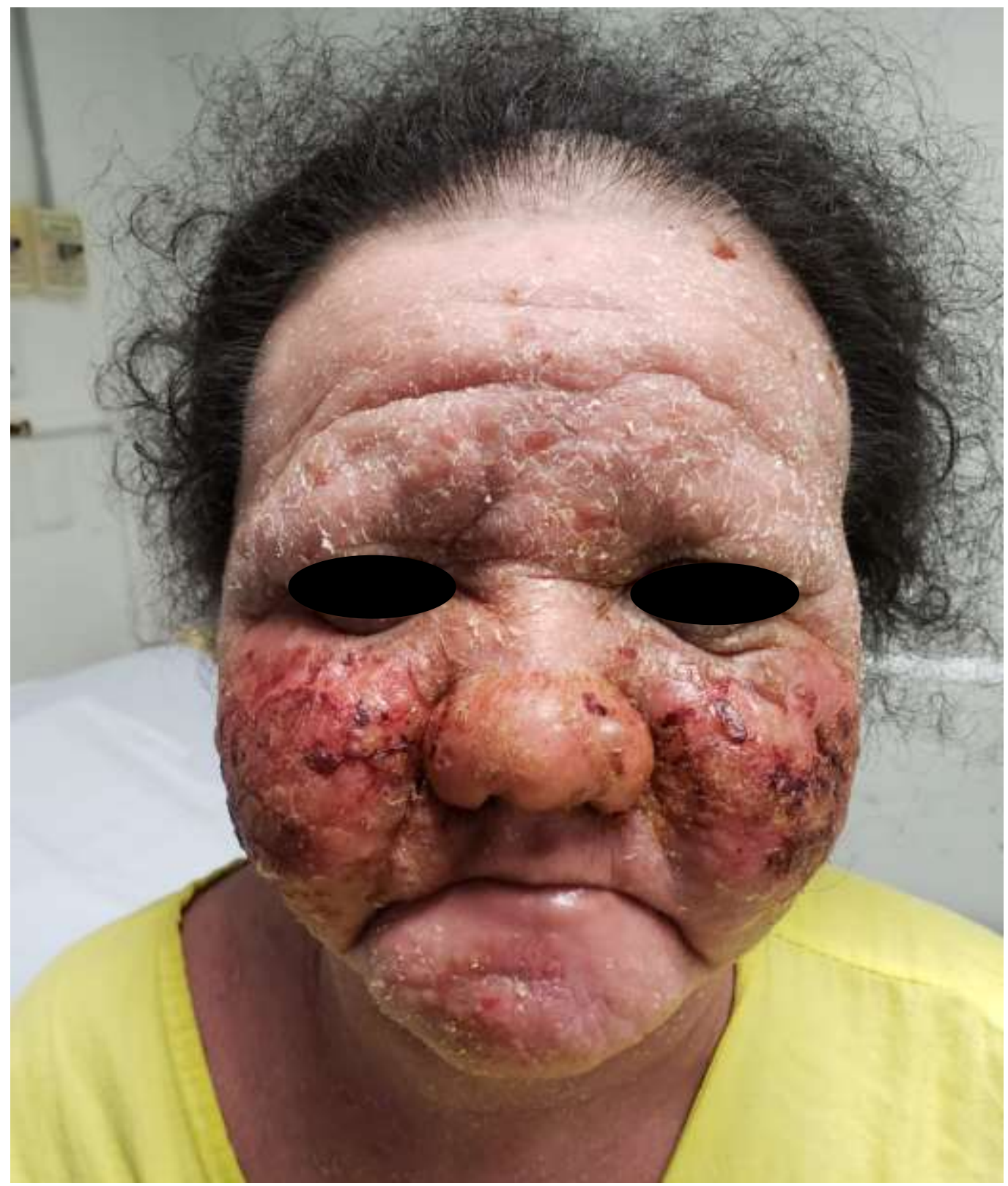

Fonte: Autores. 
Figura 2: Distrofia ungueal em pododáctilos.

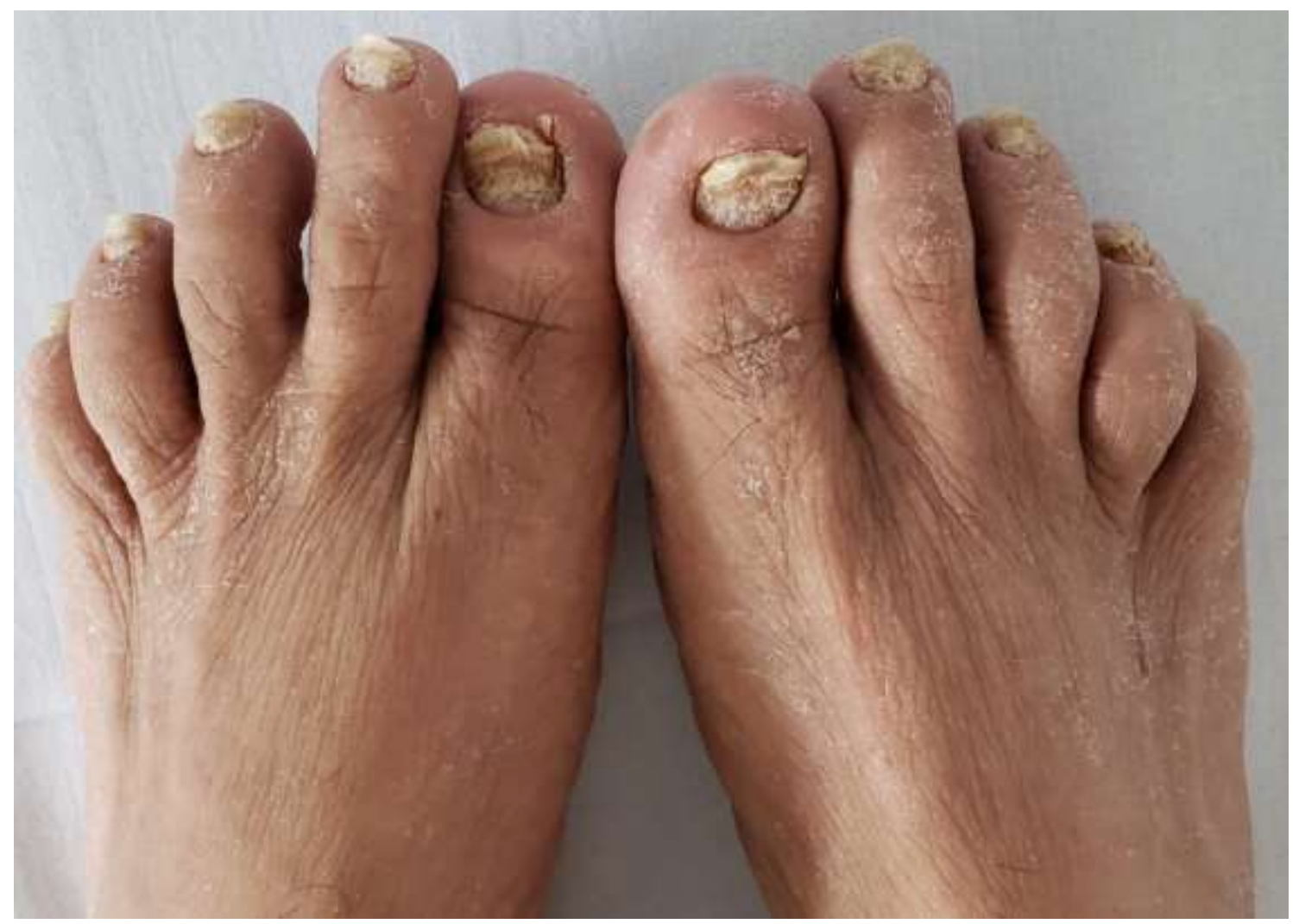

Fonte: Autores.

Realizada biópsia de pele que revelou epiderme com acantose e espongiose leves; na derme, folículos pilosos com acúmulo de mucina de localização intraepitelial acompanhados de moderado infiltrado linfocítico epidermiotrópico, com linfócitos apresentando atipias nucleares; ausência de granulomas e acantólise (Figura 3).

O infiltrado linfóide caracterizado na imunohistoquímica com positividade para CD4, CD3 e CD30. A imunofenotipagem do sangue periférico com presença de aproximadamente $15 \%$ de células linfóides $\mathrm{T}(\mathrm{CD} 3+)$ de baixa complexidade interna e pequeno tamanho, relação CD4/CD8=10, células TCD4+ (2870 células/ml) apresentam os fenótipos positivos CD45, CD3, CD4, CD5 e negativos CD2, CD8, CD19, CD20, KAPPA, LAMBDA, CD7, CD26, concluindo como doença linfoproliferativa de células T, Síndrome Sézary, com população de células (CD4/CD26-) > 30\% e população de (CD4+/CD7-) $>20 \%$.

Durante a internação, eclodiu o número de casos de COVID-19 no hospital, a paciente, além do quadro cutâneo, apresentou rinorréia, odinofagia, febre e swab de nasofaringe positivo para o novo coronavírus, não evoluindo para a síndrome respiratória aguda grave, apesar da imunossupressão da doença de base. Optou-se, assim, por conduta conservadora, mantendo-a em isolamento por 14 dias e retorno à enfermaria para seguimento clínico com encaminhamento ao serviço de hematologia em hospital universitário para início de esquema CHOP.

\section{Discussão}

SS é uma doença rara, responsável por menos de 5\% de todos os LCTT's que acometem adultos. Acomete principalmente idosos e duas vezes mais homens que mulheres (Wilcox RA, 2017). O novo coronavírus ainda está em andamento no Brasil e no mundo, com milhões de casos confirmados e milhares de óbitos, sendo uma doença com alta 
transmissibilidade e mais propensa à gravidade nos grupos vulneráveis, como idosos, portadores de doenças crônicas: hipertensos, diabéticos, renais e pulmonares obstrutivas, transplantados, obesos, coronariopatas e neoplasias malignas.

A etiopatogenia da SS ainda é incerta, porém, acredita-se que deriva das células T de memória, que se expandem clonalmente em um contexto de inflamação crônica. Essa estimulação antigênica crônica, associada a anormalidades genéticas subjacentes, podem resultar na proliferação clonal dos linfócitos atípicos. Uma grande variedade de exposições ambientais e ocupacionais foram implicados como agentes causadores (Olsen et al., 2011).

A patogênese do COVID-19 parece representar uma interação entre lesão mediada pelo vírus e resposta imune do hospedeiro, com dados experimentais sugerindo que uma resposta imune desregulada e intensa pode se associar a doenças mais graves (Chen et al, 2020).

Durante a pandemia de COVID - 19 e com base em evidências na epidemiologia, pacientes com linfomas cutâneos primários (LCP) têm risco de desenvolver infecções mais graves por COVID - 19, levando-se em consideração à idade avançada (maioria acima de 60 anos) e suas possíveis condições predisponentes subjacentes, por exemplo, hipertensão, diabetes (Guan et al., 2020). Além disso, pacientes com LCP com comportamento biológico agressivo devem ser cuidadosamente monitorados de acordo com as diretrizes para pacientes com doença neoplásica (Von Lilienfeld-Toal et al., 2020).

As células malignas presentes na SS são caracterizadas por uma expansão monoclonal de células T, geralmente com imunofenótipo $\mathrm{CD} 3+$, CD4 +, CD8-, com perda de CD5, CD7 e CD26 e aumento da razão CD4:CD8. Enquanto o CD7 é normalmente expresso em aproximadamente $90 \%$ das células T CD4 +, é total ou parcialmente perdido nas células neoplásicas em $60-70 \%$ dos casos de SS. Assim, a perda de CD7 ( $\geq 40 \%)$ e/ou CD26 ( $\geq 80 \%)$ é sensível ( $>$ 80\%) e altamente específica (100\%) para SS (Bologna 2011).

Pacientes com câncer e infecções por vírus respiratórios adquiridos na comunidade (VRAC) são propensos a coinfecções bacterianas / fúngicas, as quais são a principal causa de mortalidade associada a VRAC em pacientes com câncer (Herman Hermann 2017). Na ausência de dados mais específicos, fatores de risco potenciais para curso grave da doença de COVID-19 devem ser assumidos como para outras infecções por VRAC: imunodeficiência grave, linfopenia, neutropenia longa e profunda e idade avançada. Pacientes gravemente imunodeprimidos geralmente apresentam maior risco de desenvolver complicações em infecções por VRAC e deve-se pressupor que pacientes com câncer também estejam em risco de um curso mais grave de COVID-19.

A SS têm uma sobrevida geral correlacionada com o estágio da doença, estando a maioria dos pacientes sujeitos à maior morbi-mortalidade por infecções oportunistas devido a um sistema imunológico incompetente (Radford, Devesa, Anderson \& Toro 2009; Scarisbrick 2019). No caso em estudo, apesar da imunossupressão associada à SS, a paciente não evoluiu para forma grave da SRAG do COVID-19.

As características histopatológicas da síndrome de Sézary são praticamente indistinguíveis da MF, caracterizados por um infiltrado epidermotrópico composto de linfócitos atípicos de tamanho médio, com núcleos cerebriformes (Willemze 2019; Wilcox 2017). Variantes histopatológicas possíveis são a reação granulomatosa com depósito de mucina em folículos pilosos e a transformação em estádios avançados para linfoma de grandes células, aspectos possíveis de ocorrer nas duas doenças (Wilcox 2017).

As lesões tumorais, mais características na Síndrome de Sézary, por outro lado, mostram um infiltrado dérmico difuso, denso e profundo, com epidermotropismo diminuído ou ausente de linfócitos atípicos. Em até um terço das biópsias, os resultados são inespecíficos. Assim, a Imuno-histoquímica e a Citometria de fluxo sanguíneo são essenciais para o diagnóstico preciso do Linfoma (Olsen, et al., 2011). 
Algumas variantes histopatológicas possíveis são: reação granulomatosa, depósito de mucina em folículos pilosos e transformação em estádios avançados para linfoma de grandes células, aspectos também possíveis de ocorrer nas duas doenças (Spicknall, 2018).

No caso clínico, observamos a presença da variante foliculotrópica, com depósitos de mucina nos folículos pilosos, relacionado, muitas vezes, à presença de alopecia e madarose, características presente na paciente do caso.

Embora a etiologia não seja conhecida, a ativação da resposta imunológica T-helper tipo 2 (Th2), consistente com um perfil regulador T (também chamado de supressor T), resulta na supressão da resposta imune normal. Como resultado do viés em direção aos perfis Th2 e T regulador, há supressão de células Natural Killer e Células T CD8+, além de função anormal e diferenciação de células dendríticas, prejudicando a resposta a infecções virais e tumores (Yamashita et al., 2012; Guitart \& Magro, 2007).

Na definição dos Linfomas cutâneos, é importante avaliar o grau de envolvimento sanguíneo. Em 2002, a Sociedade Internacional de Linfomas Cutâneos propôs novos critérios para o diagnóstico de SS, incluindo um ou mais dos seguintes parâmetros: contagem absoluta de células de SS > 1000 células/mm3 no esfregaço de sangue periférico; expansão clonal de TCD4 alterados, resultando T CD4/CD8 $\geq 10$ ou células T CD4+ expandidas com imunofenótipo anormal, especificamente $\geq 40 \%$ de perda de CD7 ou $\geq 30 \%$ de perda de CD26 (Sokolowska-Wojdylo et al., 2005).

$\mathrm{Na}$ Imunofenotipagem de sangue periférico da paciente do relato de caso, obtivemos aproximadamente $15 \%$ de células linfoides $\mathrm{T}(\mathrm{CD} 3+)$, com relação $\mathrm{CD} 4 / \mathrm{CD} 8=10$, além de células T CD4+ com o fenótipo: CD45+, CD3+, CD4+, CD5+, CD2-, CD8-, CD19-, CD20-, CD7- e CD26-.

A SS se apresenta mais comumente com eritrodermia esfoliativa, intensamente pruriginosa, que antecede o diagnóstico em meses a anos. Linfadenopatia, ceratodermia palmoplantar, distrofias ungueais e alopecia são outros achados comumente associados (Kubica et al., 2012).

As fácies leoninas são raras na SS e correspondem à manifestação morfológica da infiltração dérmica difusa da face, sendo mais frequente em associação com hanseníase e LCCT's. Na maioria das vezes ocorre quando as doenças progridem sem terapia (Nassem et al., 2009).

O diagnóstico da SS caracteriza-se por uma tríade de eritrodermia (> 80\% de área corpórea comprometida), linfadenopatia e presença de células T malignas com núcleos cerebriformes (CS-células de Sérazy) no sangue periférico. O infiltrado edematoso em região cefálica pode se iniciar como lesões eczematosas em membros, intensamente pruriginosas, infiltração edematosa em face, semelhante a fácies leoninas. Comumente, a SS está associada à formação de nódulos, queratose palmoplantar, linfadenopatia múltipla, edema de membros inferiores, alopecia e distrofia ungueal.

O diagnóstico da SS continua sendo desafiador. Características clínicas da eritrodermia estão presentes em doenças inflamatórias crônicas dermatológicas, como a psoríase, pitiríase rubra pilar e dermatite atópica eritrodérmica. Assim, a história, a avaliação de exame de sangue periférico para identificar proliferação clonal de linfócitos T e histopatológico são necessários para distinguir a SS de condições clinicamente semelhantes (Spicknall, 2018).

Como a SS é uma doença rara, há uma amostra pequena para ensaios clínicos, assim, é associada à MF eritrodérmica para a busca de opções terapêuticas.

No controle do prurido na SS podem ser utilizados drogas sistêmicas como inibidores da histona desacetilase, antihistamínicos, doxepina e gabapentina. Agentes de segunda linha incluem mirtazapina e inibidores da receptação de serotonina (IRSR). Terapia tópica com emolientes e corticóides são úteis no alívio do prurido (Olsen et al., 2011).

As terapias de primeira linha para SS incluem o seguinte: fototerapia (ECP), interferon- $\alpha$ subcutâneo (IFN- $\alpha$ ); bexaroteno oral; e baixa dose de metotrexato oral, subcutâneo ou intramuscular (MTX; $\leq 100 \mathrm{mg}$ por semana) (Talpur et al., 2002; Sun et al., 1998). Combinação são possíveis, incluindo o seguinte: bexaroteno e IFN- $\alpha$; bexaroteno e ECP; IFN- $\alpha$ e ECP; 
bexaroteno, IFN- $\alpha$ e ECP; IFN- $\alpha$ e MTX de baixa dose; e IFN- $\alpha$, MTX em baixa dose e ECP (Olsen et al., 2011; Sun et al., 1998).

As terapias de segunda linha para SS incluem quimioterapia em monoterapia, inibidores da histona desacetilase, quimioterapia múlltipla (fludarabina e ciclofosfamida; ciclofosfamida, doxorrubicina, vincristina, prednisona [CHOP]) ou imunoterapia direcionada, incluindo brentuximabe (anti-CD30), alemtuzumab (anti-CD52) e mogalumizumabe (anti-CCR4) (Watanabe et al.,2014).

O transplante alogênico de células tronco, atualmente, é o único tratamento curativo potencial para os LCCT's. Entretando, está associado a alta morbimortalidade, sendo considerado apenas em pacientes jovens e relativamente saudáveis, que apresentem doença avançada (Johnson et al., 2019; Molina et al., 2005).

O prognóstico da SS é geralmente ruim com uma sobrevida, em média, de 5 anos. Os pacientes morrem frequentemente por infecções oportunistas relacionadas à imunossupressão inerente ao tratamento (Sampaio \& Rivititi, 2008).

\section{Considerações Finais}

O caso relatado e as publicações levantadas nos remetem a discussão da dificuldade do diagnóstico e manejo da SS e como novas patologias, como a COVID-19, irão influenciar a evolução, diagnóstico e terapêutica dessa patologia rara.

\section{Referências}

Bologna, J. L., Jorizzo, J. L., \& Rapini, R. P. (2012). Dermatology. Elsevier, (3a ed.), 1867-1877.

Chen, G., Wu, D., Guo, W., Cao, Y., Huang, D., Wang, H., Wang, T., Zhang, X., Chen, H., Yu, H., Zhang, X., Zhang, M., Wu, S., Song, J., Chen, T., Han, M., Li, S., Luo, X., Zhao, J., \& Ning, Q. (2020). Clinical and immunological features of severe and moderate coronavirus disease 2019. J Clin Invest. 130(5):2620. 10.1172/JCI137244

Guan, W. J., Ni, Z. Y., Hu, Y. et al. (2020). Clinical characteristics of coronavirus disease 2019 in China. N Engl J Med. $382: 1708-1720$. 10.1056/NEJMoa2002032

Guitart, J., \& Magro, C. (2007). Cutaneous T-cell lymphoid dyscrasia: a unifying term for idiopathic chronic dermatoses with persistent T-cell clones. Arch Dermatol. 143:921-932. 10.1001/archderm.143.7.921

Herman Hermann, B., Lehners, N., Brodhun, M. et al. (2017). Influenza virus infections in patients with malignancies - characteristics and outcome of the season 2014/15. A survey conducted by the Infectious Diseases Working Party (AGIHO) of the German Society of Haematology and Medical Oncology (DGHO). Eur J Clin Microbiol Infect Dis. 36: 565- 573. 10.1007/s10096-016-2833-3

Johnson, W. T., Mukherji, R., Kartan, S., Nikbakht, N., Porcu, P., \& Alpdogan, O. (2019). Allogeneic hematopoietic stem cell transplantation in advanced stage mycosis fungoides and Sezary syndrome: a concise review. Chin Clin Oncol. 8(1):12. 10.21037/cco.2018.10.03

Kubica, A. W., Davis, M. D., Weaver, A. L., Killian, J. M., \& Pittelkow, M. R. (2012). Sézary syndrome: astudy of 176 patients at Mayo Clinic. J Am Acad Dermatol. 67(6):1189-1199. 10.1016/j.jaad.2012.04.043

Liang, W., Guan, W., Chen, R. et al (2020). Cancer patients in SARS-CoV-2 infection: a nationwide analysis in China. Lancet Oncol. 21 : $335-337$. https://doi.org/10.1016/S1470-2045(20)30096-6

Molina, A., Zain, J., Arber, D.A. et al. (2005). Durable clinical, cytogenetic, and molecular remissions after allogeneic hematopoietic cell transplantation for refractory Sezary syndrome and mycosis fungoides. J Clin Oncol. 23(25):6163-6171. 10.1200/JCO.2005.02.774.

Nassem, S., Kashyap, R., Awasthi, N.P., Krishnani, N., \& Kumari, N. (2009). Sézary syndrome presenting with 'leonine facies'. Australas J Dermatol. 50(4):285-288. https://doi.org/10.1111/j.1440-0960.2009.00560

Olsen, E. A., Rook, A. H., Zic, J., Kim, Y., Porcu, P., Querfeld, C. et al. (2011). Sezary syndrome: immunopathogenesis, literature review of therapeutic options, and recommendations for therapy by the United States Cutaneous Lymphoma Consortium (USCLC). J Am Acad Dermatol 64(2):352-404. 10.1016/j.jaad.2010.08.037

Radford, P. T., Devesa, S. S., Anderson, W. F., \& Toro, J. R. (2009). Cutaneous lymphoma incidence patterns in the United States: a population-based study of 3884 cases. Blood. 113, 5064- 5073. 10.1182/blood-2008-10-184168

Sampaio, S., A. P., \& Rivititi, E. A. (2008). Dermatologia. (3a ed.), Artes Médicas, 2008. 80: 1259-1262

Scarisbrick, J. J., Quaglino, P., Prince, H. M. et al. (2019). The PROCLIPI international registry of early-stage mycosis fungoides identifies substantial diagnostic delay in most patients. Br J Dermatol. 181: 350-357. 10.1111/bjd.17258. 
Research, Society and Development, v. 10, n. 3, e58510313768, 2021

(CC BY 4.0) | ISSN 2525-3409 | DOI: http://dx.doi.org/10.33448/rsd-v10i3.13768

Spicknall, K. E. (2018). Sézary syndrome: Clinical and histopathologic features, differential diagnosis, and treatment. Seminars in Cutaneous Medicine and Surgery. 37. 10.12788/j.sder.2018.005.

Sokolowska-Wojdylo, M., Wenzel, J., Gaffal, E. et al. (2005). Absence of CD26 expression on skin-homing LCD4+ T lymphocytes in peripheral blood is a highly sensitive marker for early diagnosis and therapeutic monitoring of patients with Sezary syndrome. Clin Exp Dermatol. 30:702-706. 10.1111/j.13652230.2005.01904.x

Sun, W. H., Pabon, C., Alsayed, Y., Huang, P. P., Jandeska, S., Uddin, S. et al. (1998). Interferon-alpha resistance in a cutaneous T-cell lymphoma cell line is associated with lack of STAT1 expression. Blood. 91(2):570-6. https://pubmed.ncbi.nlm.nih.gov/9427711/

Talpur, R., Ward, S., Apisarnthanarax, N., Breuer-Mcham, J., \& Duvic, M. (2002). Optimizing bexarotene therapy for cutaneous T-cell lymphoma. J Am Acad Dermatol. 47(5):672-84. 10.1067/mjd.2002.124607

Ueda, M., Martins, R., Hendrie, P. C. et al. (2020). Managing cancer care during the COVID19 pandemic: agility and collaboration toward a common goal. $J$ Natl Compr Canc Netw; 18: 366-369. 10.6004/jncen.2020.7560

Von Lilienfeld-Toal, M., Vehreschild, J. J., Cornely, O., Pagano, P., \& Compagno FHirsch, H. H. (2020). European Hematology Association scientific working group infections in Hematology. Frequently asked questions regarding SARS-CoV2 in cancer patients: Recommendations for clinicians caring for patients with malignant diseases 2020. https://www.nature.com/articles/s41375-020-0832-y

Wang, D., Hu, B., Hu, C., Zhu, F., Liu, X., Zhang, J. et al. (2020). Clinical Characteristics of 138 Hospitalized Patients With 2019 Novel Coronavirus-Infected Pneumonia in Wuhan, China. Jama; 1-9. http://www.ncbi.nlm.nih.gov/pubmed/32031570

Watanabe, R., Teague, J. E., Fisher, D. C., Kupper, T. S., \& Clark, R. A. (2014). Alemtuzumab therapy for leukemic cutaneous T-cell lymphoma: diffuse erythema as a positive predictor of complete remission. JAMA Dermatol; 150(7):776-9. 10.1001/jamadermatol.2013.10099.

Wilcox, R. A. (2017). Cutaneous T-cell lymphoma: 2017 update on diagnosis, risk-stratification, and management. Am J Hematol. 92:1085-1102. 10.1002/ajh. 24876

Willemze, R., Cerroni, L., Kempf, W. et al. (2019). The 2018 update of the WHO-EORTC classification for primary cutaneous lymphomas. Blood. 133 (16):1703-1714. 10.1182/blood-2018-11-881268

Yamashita, T., Marques, M. E. A., Abbade, L. P. F., \& Marques, S. A. (2012). Micose fungoide e síndrome de Sézary: revisão e atualização clínica, histopatológica e imuno-histoquímica - Anais Brasileiros de Dermatologia, https://www.scielo.br/scielo.php?pid= S036505962012000600001\&script=sci_abstract\&tlng=pt 